\title{
Experimental SDR Receiver for Monitoring and Processing of Solar Radio Bursts
}

\author{
Pavel Kovar, Pavel Puricer \\ Dept. of Radioelectronics, Faculty of Electrical Engineering \\ Czech Technical University in Prague \\ Prague, Czech Republic \\ pavel.puricer@fel.cvut.cz
}

\begin{abstract}
The paper introduces a design of radio spectrography receiver that shall serve as equipment for reception, monitoring, and processing of solar radio bursts. These burst can affect operation of many radio communication and navigation services as is presented on example of GPS satellites signal degradation. The concept and design of the spectrography receiver is presented together with discussion of hardware requirements and proposed solution.
\end{abstract}

Keywords-radio solar bursts; spectrography; direct conversion; SDR; software radio

\section{INTRODUCTION}

The solar radio emission can be classified according to its manifestations to three basic components:

- $\quad$ QSR - quiet Sun radiation

- $\quad$ the slowly varying component

- emissions from disturbed Sun (radio Sun bursts)

The QSR is a steady component in time and in $\mathrm{GHz}$ bands keeps almost same value (little bellow Planck rule for black body emission). For $\mathrm{GHz}$ frequencies (decimeter wavelengths) is the radio flux density about $10^{-21}$ W.m ${ }^{-2} \mathrm{~Hz}^{-1}$ depending on frequency [1]. The main source is a standard thermal emission of various solar atmosphere layers. It can be considered as a main component of a background noise and used for example for radio spectrograph calibration according to formula

$$
F_{\text {cal }}(f)=\frac{Q S R_{\text {teor }}(f)}{Q S R_{\text {meas }}(f)-N(f)}\left[F_{\text {meas }}(f)-N(f)\right]
$$

where $F_{\text {cal }}$ is recalibrated flux of measured radio burst data, $Q S R_{\text {teor }}$ is theoretical spectrum of QSR background emission, $Q S R_{\text {meas }}$ is measurement of the QSR by the used equipment during the quiet state of the Sun (i.e. outside the time of bursts), $F_{\text {meas }}$ measured flux of radio burst data, and $N$ is internal noise of equipment measured with the antenna pointed in the other direction than to the Sun. The QSR processing is not the main focus of the designed spectrograph application but we will use it for receiver calibration.

The slowly varying component has a form of light modulation of received QSR signal. It is related to presence of active spots on Sun, where high density plasma is captured, and this accumulation leads to excessive radiation. Typical time dependence is coherent with synodic rotation period of the Sun (about 26.24 days for equatorial based rotation)

Radio Sun bursts are the short time (in order of tens of seconds up to several hours) increase of the signal level, very often band limited, and with time varying waveform in order of about milliseconds. The relative increase of the signal level is about 3 to 4 orders more with respect to QSR level. The observation, recording, and processing of these bursts is main purpose of the proposed spectrograph design.

\section{IMPACT OF SOLAR BURSTS TO RADIOSIGNAL RECEPTION}

The solar radio burst cover wide range of frequencies, therefore they can affect operation of various radiocommunication and radionavigation services.

One of the examples can be impact of solar radio bursts in L band to operation of GNSS (Global navigation Satellite Systems). Already during preliminary tests of GPS there was predicted that such bursts can degrade performance of GPS system if they will have sufficiently high flux and RHCP (Right Hand Circular Polarization) that GPS antennas are designed to receive [2]. Further tests have shown observable decrease of $\mathrm{C} / \mathrm{N}_{0}$ (Carrier to Noise Ratio) of GPS satellite signals that can drop below level required for signal acquisition and tracking [3].

\footnotetext{
Figure 1. Comparison of C/N0 for GPS PRN12 in two sidereal days
}

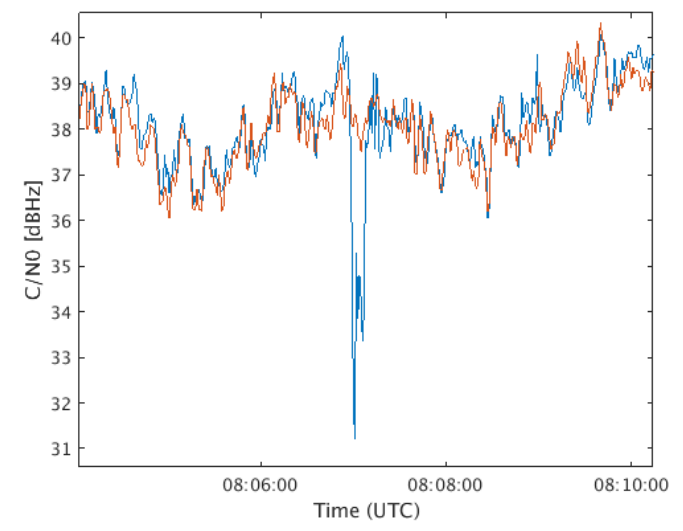


An example of $\mathrm{C} / \mathrm{N}_{0}$ dropout measured at monitoring and reference station of Czech Technical University, Faculty of Electrical Engineering, Prague, is shown at Fig. 1. There is record of GPS satellite PRN13 for two consecutive sidereal days, $25^{\text {th }}$ and $26^{\text {th }}$ June 2015, where blue curve shows observable drop of signal strength correlated with data about sun radio burst provided by AsU AS CR in Ondrejov. This radio burst was however quite small, the increase of flux was only by one order. So for big bursts with flux increase by 3 or 4 orders we can expect drop of $\mathrm{C} / \mathrm{N}_{0}$ by tens of decibels.

\section{THE RADIOSPECTROSCOPY RADIO RECEIVER}

\section{A. The case study of radiospectroscopy radio} receiver

The design of receiver is based on conditions related to current state of spectroscopy observations executed at Ondrejov AI ASCR observatory. According to [4], the measurements in the decimeter band are realized by RT5 spectrograph (Fig.2). RT5 spectrograph is sequentially tuned spectrograph in band 0.8 to $2 \mathrm{GHz}$ fed by dish antenna with diameter $9.5 \mathrm{~m}$ on azimuthal mount automatically steered to Sun. It provides observations on one chosen polarization with resolution of $10 \mathrm{~ms}$ at 256 channels of $4 \mathrm{MHz}$ width per one channel.

Figure 2. RT5 spectrograph installation in AI ASCR Ondrejov

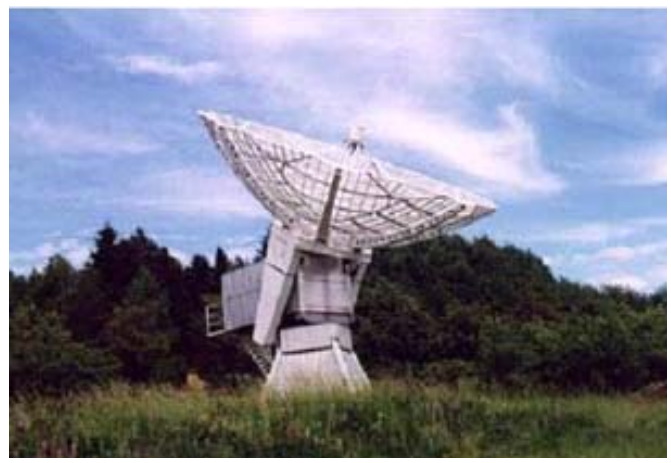

The main drawback of current solution is the limitation of time resolution caused by sequential tuning. The $10 \mathrm{~ms}$ shared between 256 consecutively tuned channels (channel sweep) means limitation of integration time to about $10^{-5} \mathrm{~s}$ per channel. For such signal integration time there is still present significant background noise caused by quiet Sun. The increase of integration time to remove this background noise impact is however for current sequential approach not possible without loss of time resolution of measurement. Therefore the solution based on parallel FFT computation of whole observed band is chosen for proposed design.

The current frequency resolution is not a limiting parameter because the most narrowband artifacts of solar bursts in band $1-2 \mathrm{GHz}$ are dm spikes, which have about $20 \mathrm{MHz}$ bandwidth [5].
B. The design of receiver for continuous parallel spectrum observation in band 1-2 GHz

The high-level requirements on the spectrograph are:

- Operation bandwidth $1-2 \mathrm{GHz}$

- Maximal resolution bandwidth $4 \mathrm{MHz}$

- Optimal resolution bandwidth $1 \mathrm{MHz}$

- Dynamic range

$>60 \mathrm{~dB}$

- Time resolution

$1 \mathrm{~ms}$

As the amplitude characteristic can be calibrated on the quite sun radiation, there are not special requirements on the amplitude ripple, the amplitude ripple $<6 \mathrm{~dB}$. The phase of the signal is not investigated; therefore, the phase distortion of the signal is not a problem. The sensitivity of the spectrograph is a critical parameter. The spectrograph has to implement optimal signal processing methods, i.e. all frequency bins have to be investigated in parallel continually in time and averaged for $1 \mathrm{~ms}$ integration time. The sequential observation is not allowable.

On the other hand, the splitting of the frequency range onto any number of hardware subchannels is possible.

The evaluation of the measurement data will be done by the experienced researcher that is able to distinguish between radio interference and sun radioactivity. The corruption of several spectral bins by the spurious response caused by the spectrograph circuits is tolerated but their level cannot saturate ADC converter.

During the conceptual design of the spectrograph the following receiver architecture was considered:

The first option is a tuned RF radio frequency receiver that just amplifies and filters the signal coming from the antenna. This architecture uses RF sampling method for analog to digital conversion. This architecture was rejected due to the requirement on sampling in the first zone. The sampling frequency is very high, out of the technical capability of the current ADC circuits.

Single conversion superheterodyne receiver was investigated as a second possibility. The problem is the realization of the intermediated filters due to the extremely wide bandwidth that complicates filter realization. The second serious problem is overlapping of the spectra of received signal and intermediated signal.

The promising results are expected from the direct conversion receiver. The receiver requires half sampling frequency in comparison to the receivers that implement baseband or radio frequency sampling. On the other hand, the analog to digital converter have to process two I\&Q signals as the baseband representation of the radio frequency signal is a complex value. The whole frequency band can be processed in single channel thanks to the high sampling frequency of today's converters. The problem is with the cost of this solution because the 
price of the converter as well as FPGA that controls the interface with such converter is very high. The preliminary analyses show that the price of the spectrograph based on this architecture starts from 30000 USD.

The further analyses had shown that the price can by reduced by splitting of the operating bandwidth on to four channel of bandwidth $250 \mathrm{MHz}$ as the price of the analog to digital converter as well as FPGA grows much faster than the bandwidth. Therefore, the solution of direct conversion receiver with several parallel channels monitoring concatenated bandwidths was chosen as is depicted on Fig. 3.

Figure 3. Block scheme of the receiver

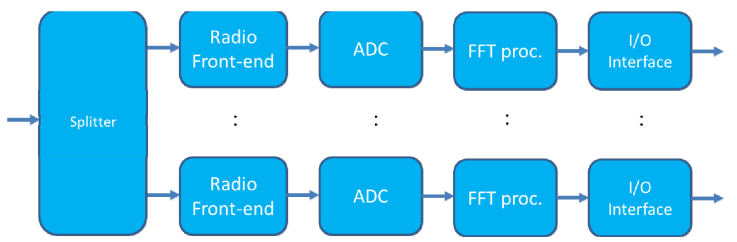

The problem of the direct conversion receiver is crosstalk between upper and lower sidebands caused by the phase and amplitude unbalance of I and Q receiver branches. This unbalance can be compensated by the feedbacks that are analyzed in the next paragraph.

\section{Distortion of receiver sidebands caused by $I / Q$ imbalance}

Since we use the direct sampling of in-phase (I) and quadrature $(\mathrm{Q})$ components of the signal, there is an important impact of distortion of sidebands caused by amplitude and phase imbalance in I and Q branches. This impact can be described by suppression ratio of sideband distortion and according to [6] can be expressed by

$$
S D S R=10 \log \left(\frac{1+\varepsilon_{R}^{2}+\varepsilon_{R}^{2} \tan ^{2}\left(\Delta \phi_{R}\right)}{\varepsilon_{R}^{2}+\tan ^{2}\left(\Delta \phi_{R}\right)}\right)
$$

Figure 4 shows sideband distortion suppression according to [6] where values were depicted for result better than $20 \mathrm{~dB}$ (the dark blue mesh reference plane).

Figure 4. Sideband distortion suppresion

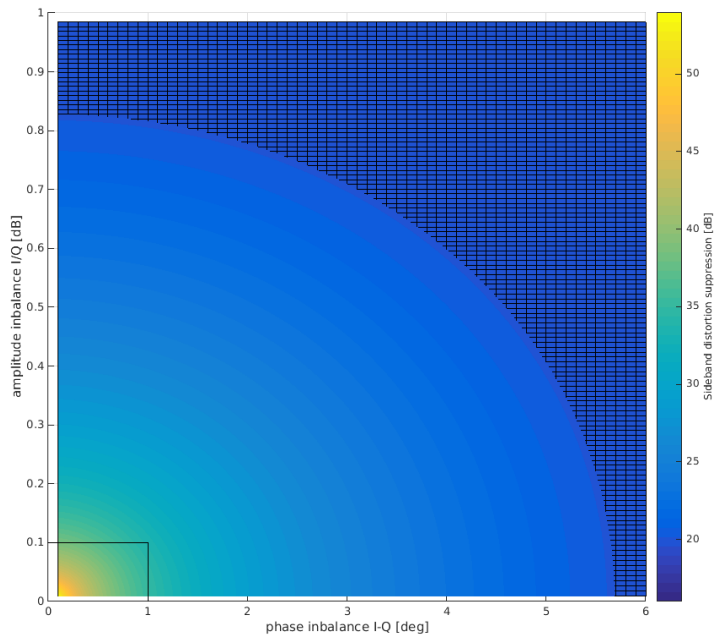

The ADRF6820 circuit, which was chosen for the I/Q demodulation, provides amplitude imbalance better than $0.1 \mathrm{~dB}$ and phase imbalance better than $1^{\circ}$. These limits are in the graph expressed by rectangular region in lower-left corner. The remaining mutual distortion of I and Q branches will then be adaptively compensated in FPGA.

The designed receiver will be equipped with three compensation feedback solutions:

Feedback for compensation of the DC offsets of I and $\mathrm{Q}$ receiver branches. The detector $d_{D C}$ of this feedback will investigate the DC level of the I and Q analog to converter channels

$$
d_{D C}=\sum_{N} I_{N}-\sum_{N} Q_{N}
$$

Feedback for amplitude unbalance compensation that detector $d_{\text {amp }}$ will compare the signal level of the I and $\mathrm{Q}$ outputs and control the gain of one of them with the aim to balance amplitude response.

$$
d_{\text {amp }}=\sum_{N} I_{N}^{2}-\sum_{N} Q_{N}^{2}
$$

Feedback for the phase unbalance compensation. The phase unbalance detector $d_{p}$ is

$$
d_{p}=\frac{\sum_{N} I_{N} Q_{N}}{\sum_{N} I_{N}^{2}+\sum_{N} Q_{N}^{2}}
$$

The values $I_{N}$ and $Q_{N}$ are signal samples in I and Q branches.

\section{Receiver Interface}

For the choice of proper interface, the bit rate considerations have to be taken into account.

The one channel of the receiver will use 1024 frequency bins in the channel, each sample will use 24 bit resolution which together with desired $1 \mathrm{~ms}$ integration yields in $24.6 \mathrm{Mbit} / \mathrm{s}$. Therefore the interface conforming of at least USB High speed performance has to be considered. The example can be an FT2232H - Hi-Speed Dual USB UART/FIFO IC providing FIFO of $40 \mathrm{Mbit} / \mathrm{s}$. To be able to synchronize measurements from several channels together, the timestamp for each set of FFT outputs will be taken from external GPS 1PPS signal decimated to 10 s timestamp.

\section{E. The Impact of Inband Interference}

The designed receiver will process signals in $1 \mathrm{GHz}$ bandwidth with expected dynamics from QSR up to $50 \mathrm{~dB}$ higher. The flux power density of QSR [1] is then $70 \times 10^{-22} \mathrm{~W} / \mathrm{m}^{2} \mathrm{~Hz}^{-1}$ over $10^{9} \mathrm{~Hz}$ bandwidth which yields $7 \times 10^{-12} \mathrm{~W} / \mathrm{m}^{2}$. The saturation of the receiver then comes for power flux of about $7 \times 10^{-7}$ $\mathrm{W} / \mathrm{m}^{2}$. We have then considered possibility of inband interference by other radio service that can saturate receiver and destroy the measurement.

The mobile phone with its peak power of $2 \mathrm{~W}$ excitates in the distance of $1000 \mathrm{~m}$ the power flux 
density of $1.59 \times 10^{-7} \mathrm{~W} / \mathrm{m}^{2}$ and in the distance of 100 $\mathrm{m}$ density of $1.59 \times 10^{-5} \mathrm{~W} / \mathrm{m}^{2}$. Therefore it is necessary to assure that receiver antenna have to avoid pointing to mobile phone or BTS, otherwise it will spoil the measurement in whole evaluated band.

The similar problem occurs with airplanes crossing the path antenna - Sun. Most of them are equipped with Mode S transponder operating on frequency 1090 $\mathrm{MHz}$ (i.e. in observed band). According to [7] the output power of such transponder is $500 \mathrm{~W}$ which in typical flight height $10000 \mathrm{~m}$ provides power flux density of $3.9 \times 10^{-7} \mathrm{~W} / \mathrm{m}^{2}$ and in case of descent to approach a runway with typical height of $1000 \mathrm{~m}$ means power flux density again in at levels of $10^{-5}$ $\mathrm{W} / \mathrm{m}^{2}$. This means to monitor flight paths of airplanes and stop the measurement in case that their path will cross line of sight of spectrograph.

\section{CONCLUSIONS}

The proposed receiver is designed to cover whole observable range of 0.8 to $2 \mathrm{GHz}$ with sufficient time and frequency bins resolution so it can serve as versatile monitoring tool not only for radio solar bursts observation but thanks to combination of several parallel channels with narrower bandwidth it can be easily adjusted to different bandwidth combinations. It will be used together with GNSS monitoring station also for evaluation of possible impact of such solar activity on performance of GNSS which is at present, when we expect increase of solar activity and with massive infiltration of GNSS based services into various areas of common life, the question of increasing importance. The performance of receiver will be comparable with similar projects, e.g. Callisto [8], used for monitoring in HF band or AWESOME [9], monitoring in VLF band.

\section{ACKNOWLEDGMENT}

The preparation of this publication was supported by OP RDE, MEYS, Czech Republic under the project CRREAT, CZ.02.1.01/0.0/0.0/15 003/0000481. and by project of the Czech Technical University SGS16/166/OHK3/2T/13.

\section{REFERENCES}

[1] Benz, A.O. Quiet and Slowly Varying Radio Emissions of the Sun. The Landolt-Boernstein Database, LB VI/4B 4.1.1.6. 2009. http://dx.doi.org/10.1007/978-3-540-88055-4

[2] Klobuchar, J. A., Kunches J. M., and Van Dierendonck A. J., Eye on the ionosphere: Potential solar radio burst effects on GPS signal to noise, GPS Solutions, 3, $69-71,1999$. doi:10.1007/PL00012794.

[3] Cerruti, A. P., Kintner Jr. P. M., Gary D. E., Mannucci A. J., Meyer R. F., Doherty P., and Coster A. J., Effect of intense December 2006 solar radio bursts on GPS receivers, Space Weather, 6, S10D07, 2008. doi:10.1029/2007SW000375

[4] Barta, M. The comments to planned reconstruction of radio spectrographs at AsU AV CR in Ondřejov, research report, 9 pages, AsU AV CR, June 2016.unpublished.

[5] Yan, Y., Liu, Y., Chen, Z., Fu, Q., Tan, C., Wang, S., Occurrence of Solar Radio Burst Fine Structures in 1-7.6 GHz Range Associated with CME Events. Coronal and Stellar Mass Ejections, IAU Symposium Proceedings of the International Astronomical Union 226, Held 13-17 September, Beijing, edited by K. Dere, J. Wang, and Y. Yan. Cambridge: Cambridge University Press, 2005., pp.101-107

[6] Horlin F., Bourdoux A., Digital Compensation for Analog Front-Ends. England: A John Wiley \& Sons, Ltd. Publication, 2008

[7] RTCA, Inc.,DO-260B Minimum Operational Performance Standards for $1090 \mathrm{MHz}$ Extended Squitter Automatic Dependent Surveillance - Broadcast (ADS-B) and Traffic Information Services - Broadcast (TIS-B), 2009.

[8] Nosengo N., Global solar observatory flares into life. Homebuilt e-CALLISTO network provides real-time data on Sun's radio emissions. Nature, Published online 17 February 2011, DOI:10.1038/news.2011.97.

[9] Cohen, M. B., U. S. Inan, E. W. Paschal (2010), Sensitive broadband ELF/VLF radio reception with the AWESOME instrument, IEEE Trans. Geosc. Remote Sensing, Vol 48, Issue 1, Pages 3-17, doi:10.1109/TGRS.2009.2028334. 